\title{
Synchrony-induced modes of oscillation of a neural field model
}

\author{
Jose M. Esnaola-Acebes, ${ }^{1}$ Alex Roxin, ${ }^{2}$ Daniele Avitabile, ${ }^{3}$ and Ernest Montbrió ${ }^{1}$ \\ ${ }^{1}$ Center for Brain and Cognition, Department of Information and Communication Technologies, \\ Universitat Pompeu Fabra, 08018 Barcelona, Spain \\ ${ }^{2}$ Centre de Recerca Matemàtica, Campus de Bellaterra, Edifici C, 08193 Bellaterra (Barcelona), Spain \\ ${ }^{3}$ Centre for Mathematical Medicine and Biology, School of Mathematical Sciences, University of Nottingham, \\ Nottingham NG2 7RD, United Kingdom
}

(Received 28 August 2017; published 13 November 2017)

\begin{abstract}
We investigate the modes of oscillation of heterogeneous ring networks of quadratic integrate-and-fire (QIF) neurons with nonlocal, space-dependent coupling. Perturbations of the equilibrium state with a particular wave number produce transient standing waves with a specific temporal frequency, analogously to those in a tense string. In the neuronal network, the equilibrium corresponds to a spatially homogeneous, asynchronous state. Perturbations of this state excite the network's oscillatory modes, which reflect the interplay of episodes of synchronous spiking with the excitatory-inhibitory spatial interactions. In the thermodynamic limit, an exact low-dimensional neural field model describing the macroscopic dynamics of the network is derived. This allows us to obtain formulas for the Turing eigenvalues of the spatially homogeneous state and hence to obtain its stability boundary. We find that the frequency of each Turing mode depends on the corresponding Fourier coefficient of the synaptic pattern of connectivity. The decay rate instead is identical for all oscillation modes as a consequence of the heterogeneity-induced desynchronization of the neurons. Finally, we numerically compute the spectrum of spatially inhomogeneous solutions branching from the Turing bifurcation, showing that similar oscillatory modes operate in neural bump states and are maintained away from onset.
\end{abstract}

DOI: 10.1103/PhysRevE.96.052407

\section{INTRODUCTION}

Since the pioneering work of Wilson-Cowan [1], Amari [2,3], and Nunez [4], continuum descriptions of neuronal activity have become a powerful modeling tool in neuroscience [5-10]. Given that the number of neurons in a small region of cortex is very large, these descriptions consider neurons to be distributed along a continuous spatial variable and the macroscopic state of the network to be described by a single, space-dependent, firing rate variable. The resulting neural field model (NFM) generally has the form of a continuous first-order integrodifferential equation, greatly facilitating the computational and mathematical analysis of the dynamics of large neuronal networks.

NFMs do not generally represent proper mathematical reductions of the mean activity of a network of spiking neurons. Nevertheless, NFMs have proven to be remarkably accurate in qualitatively capturing the main types of dynamical states seen in networks of large numbers of asynchronous spiking neurons. For example, it is well known that, in local networks of spiking neurons, differences between excitatory and inhibitory neurons can lead to oscillations [11-13]. The generation of these oscillations does not depend on the spatial character of the network and hence can be observed in nonspatially dependent firing rate models [12]. When the pattern of synaptic connectivity depends on the distance between neurons, NFMs show that these differences between excitation and inhibition can lead to the emergence of oscillations and waves $[3,14]$. Similar patterns can also be found in NFMs with spatially dependent delays-modeling the effect of the finite velocity propagation of action potentials $[1,15]$ - as a great deal of theoretical work indicates, see e.g., Refs. [16-23].

In some cases the spatiotemporal dynamics of NFMs has been directly compared to that observed in analogous networks of spiking neurons [24-26]. In this work it was found that non-space-dependent delays predict the existence of many of the spatiotemporal patterns observed in asynchronous networks of spiking neurons with nonlocal, space-dependent interactions. The success of NFMs in describing these patterns depends crucially on the spiking activity being highly asynchronous. In fact, it is well known that neural field descriptions fail to describe states characterized by a high degree of spike synchronization, see, e.g., Ref. [27].

Here we report a spatiotemporal dynamical feature of heterogeneous networks of spiking neurons with nonlocal interactions that, to the best of our knowledge, have been so far unexplored. We show that ring networks of spiking neurons display a number of discrete modes of oscillation, resembling those of a tense string. These modes are exclusively due to transient episodes of synchronous spiking and not due to the different time scales between excitation and inhibition or to the presence of any propagation or synaptic delay.

Traditional NFMs do not describe these synchrony-induced oscillations. Therefore, to investigate and characterize them, we apply a recent method to derive the firing rate equations of a globally coupled heterogeneous population of quadratic integrate-and-fire (QIF) neurons [28]. This method, based on the so-called Ott-Antonsen theory [29-31], leads to an exact macroscopic description of the network in terms of two macroscopic variables: the mean firing rate and the mean membrane potential. The resulting mean-field model exactly describes any state of the system, including synchronous states. Here we extend the local firing rate model in Ref. [28] to include nonlocal, instantaneous interactions. The resulting neural field model for heterogeneous QIF neurons (QIFNFM) clearly displays the synchrony-induced oscillatory modes observed in simulations of spiking neurons. We then thoroughly investigate the QIF-NFM by means of both a linear 


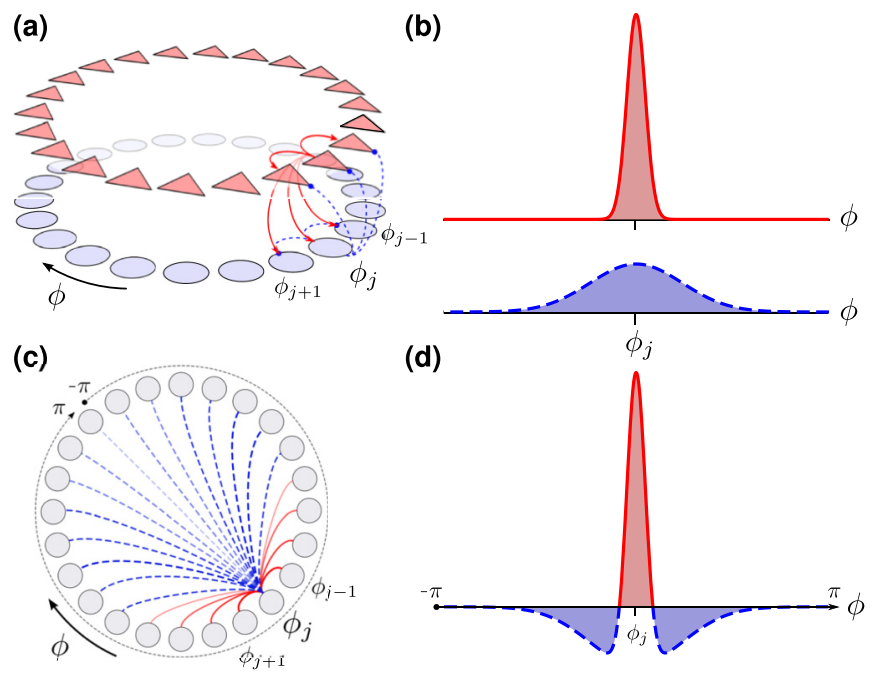

FIG. 1. Schematic representation of the ring network and coupling architecture under study. Panel (a) shows $N$ excitatory (red triangles) and $N$ inhibitory (blue circles) neurons arranged on a ring. The location of neurons is parameterized by the angular variable $\phi_{j}=$ $\frac{2 \pi j}{N}-\pi, j=1, \ldots, N$. Red (solid) and blue (dashed) lines indicate synaptic connections between neuron pairs $\left(\phi_{j}, \phi_{k}\right)$. An example of the excitatory and inhibitory space-dependent connectivity kernels Eqs. (1) are shown in panel (b) where the abscissa represents the distance $\left|\phi_{k}-\phi_{j}\right|$ between neurons $j$ and $k$. Panel (c) represents an effective model in which pairs of excitatory/inhibitory neurons located at a certain location $\phi_{k}$ are modeled as single neurons. The effective pattern of synaptic connectivity is obtained subtracting the inhibitory pattern from the excitatory one, as show in panel (d).

and nonlinear stability analysis of the spatially homogeneous state. The analysis reveals the presence of an infinite number of oscillation modes, linked to the Fourier components of the spatial pattern of synaptic connections. The analysis also shows that all modes decay to the unpatterned state with the same rate, which depends on the degree of heterogeneity in the network. Finally, we investigate the spectrum of the spatially inhomogeneous solutions of the QIF-NFM and find similar oscillatory modes also linked to transient episodes of spike synchronization.

\section{SYNCHRONY-INDUCED MODES OF OSCILLATION IN NETWORKS OF QIF NEURONS}

Figure 1(a) shows a schematic representation of the spiking neuron network under investigation. The model consists of $N$ excitatory (red) and $N$ inhibitory (blue) neurons evenly distributed in a ring and characterized by the spatial discrete variables $\phi_{j} \in[-\pi, \pi)$ with $\phi_{j}=\frac{2 \pi j}{N}-\pi, j=1, \ldots, N$, as shown in Fig. 1(a). Any neuron in the network interacts with all the other neurons via the distance-dependent coupling function $J_{j k}^{e, i}=J^{e, i}\left(\left|\phi_{j}-\phi_{k}\right|\right)$, where indices $e, i$ denote excitatory and inhibitory connections, respectively. The synaptic projections of the $j$ th excitatory and inhibitory neurons (located at at $\phi_{j}$ ) to other two nearby neurons are also schematically represented in Fig. 1(a).

The ring architecture of the network allows one to express the excitatory and inhibitory connectivity patterns in Fourier series as

$$
J^{e, i}(\phi)=J_{0}^{e, i}+2 \sum_{K=1}^{\infty} J_{K}^{e, i} \cos (K \phi) .
$$

Figure 1(b) shows a particular synaptic connectivity pattern in which excitatory neurons form strong, short-range connections, whereas inhibitory projections are weaker and wider. The state of the excitatory $(e)$ and inhibitory $(i)$ neurons is determined by the (dimensionless) membrane potentials $\left\{v_{j}^{e, i}\right\}_{j=1, \ldots, N}$, which are modeled using the QIF model [32,33]

$$
\tau \frac{d v_{j}^{e, i}}{d t}=\left(v_{j}^{e, i}\right)^{2}+I_{j}^{e, i}, \quad(+ \text { resetting rule }),
$$

where $\tau$ is the cell's membrane time constant and $v_{r}$ and $v_{p}$ correspond to the reset and peak potentials of the QIF neurons, respectively - in numerical simulations we consider $\tau=20 \mathrm{~ms}$. The QIF neuron has two possible dynamical regimes depending on the (dimensionless) input current $I_{j}^{e, i}$. If $I_{j}^{e, i}<0$, then the neuron is in the excitable regime, while for $I_{j}^{e, i}>0$ the neuron is in the oscillatory regime. In the excitable regime, an initial condition $v_{j}^{e, i}(0)<\sqrt{-I_{j}^{e, i}}$, asymptotically approaches the resting potential $-\sqrt{-I_{j}^{e, i}}$. On the other hand, initial conditions above the excitability threshold, $v_{j}^{e, i}(0)>\sqrt{-I_{j}^{e, i}}$, lead to an ubounded growth of the membrane potential. Specifically, if $v_{j}^{e, i}(0) \gg \sqrt{I_{j}^{e, i}}$, then the membrane potential reaches infinity approximately after a time $\tau / v_{j}^{e, i}(0)$. In practice, to avoid this divergence, we consider the following resetting rule: When the neuron's membrane potential $v_{j}^{e, i}$ reaches a certain peak value $v_{p} \gg 1$, the neuron is reset to a the new value $v_{r}=-v_{p}$ after a refractory period $2 \tau / v_{p}$. On the other hand, if $I_{j}^{e, i}>0$, then the neuron is in the oscillatory regime and needs to be reset periodically. If $v_{p} \gg 1$, then the frequency of the oscillatory neurons is approximately $f_{j}=\sqrt{I_{j}} /(\tau \pi)$. Finally, the current $I_{j}^{e, i}$ is defined as

$$
I_{j}^{e, i}=\eta_{j}^{e, i}+\tau S_{j}^{e}(t)+\tau S_{j}^{i}(t)+P_{j}^{e, i}(t) .
$$

Here $\eta_{j}^{e, i}$ is a constant external current, which varies from neuron to neuron. The terms $P^{e, i}(t)$ are time-varying common inputs, and $S_{j}^{e, i}(t)$ are the mean excitatory (positive) and inhibitory (negative) synaptic activities representing all the weighted inputs received by neuron $j$ due to spiking activity in the network:

$$
S_{j}^{e, i}(t)= \pm \sum_{k=1}^{N} \frac{J_{j k}^{e, i}}{2 \pi N} \sum_{l \backslash t_{k}^{l}<t} \frac{1}{\tau_{s}} \int_{t-\tau_{s}}^{t} d t^{\prime} \delta^{e, i}\left(t^{\prime}-t_{k}^{l}\right),
$$

where $\tau_{s}$ represents the synaptic processing time and $t_{k}^{l}$ is the time of the $l$ th spike of the excitatory/inhibitory $k$ th neuron. Positive and negative signs correspond $S_{j}^{e}$ and to $S_{j}^{i}$, respectively.

We performed numerical simulations of the QIF model Eqs. (2) and (3) for a network of heterogeneous neurons, see Fig. 2, and Appendix D for details of the numerical simulations. In all cases, the system is initially at a spatially homogeneous state (SHS). At time $t=50 \mathrm{~ms}$, a brief (10 ms) and small current pulse $P_{j}^{e}$ is applied either to all excitatory 
(a)

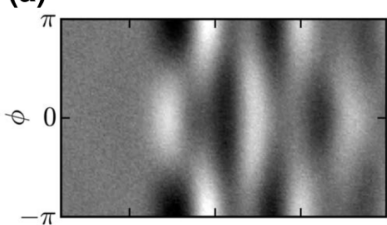

(c)

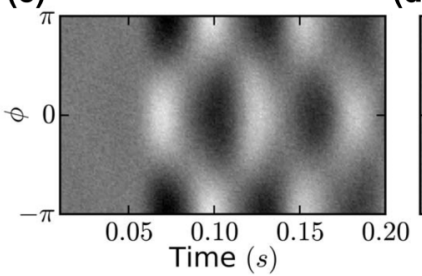

(d)

(b)
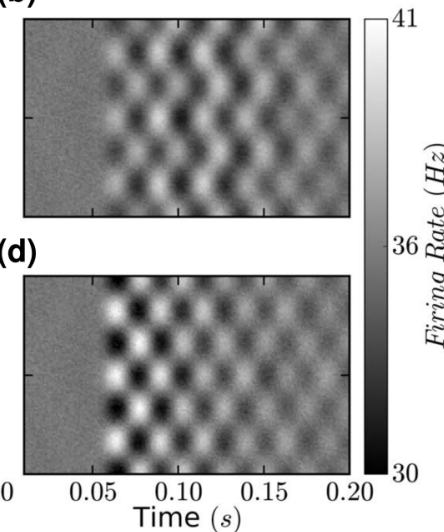

FIG. 2. Transient episodes of spike synchrony in heterogeneous ring networks of $N=2.5 \times 10^{5}$ excitatory and $N=2.5 \times 10^{5}$ inhibitory QIF neurons, Eqs. (2) and (3), as a result of spatially inhomogeneous perturbations applied at time $t=0.05$. In panels (a) and (b) only excitatory neurons are perturbed. In panels (c) and (d) all neurons are perturbed. In panels (a) and (c) the perturbation has wave number $K=1$; in panels (b) and (d) the perturbation has wave number $K=3$. Other parameters are $\Delta=1, \tau=20 \mathrm{~ms}$, and $\bar{\eta}=5$. All Fourier components of the connectivity Eq. (1) are $J_{K}^{e, i}=0$, except $J_{0}^{e}=23, J_{1}^{e}=10, J_{2}^{e}=7.5, J_{3}^{e}=-2.5, J_{0}^{i}=23$.

neurons [Figs. 2(a) and 2(b)] or to both excitatory and inhibitory neurons. The left and right panels show perturbations of the first spatial modes, respectively-see Appendix D for the specific form of the perturbations. Note that, after the perturbation, the system decays to the homogeneous state showing oscillations, which resemble standing waves. Note that the frequency of these oscillations is different for each mode, while the decay rate is similar in the two cases. We also performed simulations of networks of QIF neurons (i) with quenched Gaussian heterogeneity (ii) subject to independent Gaussian noise processes and found similar results (not shown). To the best of our knowledge, these oscillations have not yet been investigated in the literature.

\section{NEURAL FIELD MODEL FOR QUADRATIC INTEGRATE AND FIRE NEURONS}

In the following, we aim to investigate the nature and origin of the spatiotemporal patterns shown in Fig. 2. To analyze them, we derive the NFM corresponding to the thermodynamic $(N \rightarrow \infty)$ and continuum limits of the network of QIF neurons [Eqs. (2) and (3)]. In addition we also take the limit $v_{p} \rightarrow \infty$, so that the QIF model (2) is equivalent to the so-called theta-neuron model $[32,33]$. This leads to an exact neural field model for a network of QIF neurons (QIF-NFM) The detailed derivation is performed in Appendix A and closely follows that of Ref. [28]. The reduction in dimensionality is achieved considering that the currents $\eta^{e, i}$ — which, after performing the thermodynamic limit, become continuous random variablesare distributed according to a Lorentzian distribution of half-width $\Delta$ and centered at $\bar{\eta}$,

$$
g\left(\eta^{e, i}\right)=\frac{\Delta}{\pi} \frac{1}{\left(\eta^{e, i}-\bar{\eta}\right)^{2}+\Delta^{2}} .
$$

The QIF-NFM is

$$
\begin{aligned}
\tau \frac{\partial R^{e, i}}{\partial t} & =\frac{\Delta}{\pi \tau}+2 R^{e, i} V^{e, i} \\
\tau \frac{\partial V^{e, i}}{\partial t} & =\left(V^{e, i}\right)^{2}+\bar{\eta}-\left(\pi \tau R^{e, i}\right)^{2}+\tau S(\phi)+P^{e, i}(\phi, t)
\end{aligned}
$$

and exactly describes the time evolution of the mean firing rate $R^{e, i}(\phi)$ and the population's mean membrane potential $V^{e, i}(\phi)$ of the excitatory and inhibitory populations at any location $\phi$ of the ring - to facilitate the notation we have avoided explicitly writing the dependence of these variables on $\phi$. In the limit of instantaneous synapses, $\tau_{s} \rightarrow 0$ in Eqs. (4), the excitatory and inhibitory contributions of the mean field $S(\phi)=S^{e}(\phi)+$ $S^{i}(\phi)$ reduce to $S^{e, i}(\phi)= \pm \frac{1}{2 \pi} \int_{-\pi}^{\pi} J^{e, i}\left(\phi-\phi^{\prime}\right) R^{e, i}\left(\phi^{\prime}\right) d \phi^{\prime}$.

\section{A. Effective QIF-NFM}

The analysis of the QIF-NFM Eq. (6) is greatly simplified considering that excitatory and inhibitory neurons have identical single-cell properties. This scenario is schematically represented in Figs. 1(c) and 1(d). If we set $P^{e}(\phi, t)=P^{i}(\phi, t)=$ $P(\phi, t)$, and restrict our attention to solutions of Eqs. (6) satisfying $R^{e}(\phi, t)=R^{i}(\phi, t) \equiv R(\phi, t)$ and $V^{e}(\phi, t)=V^{i}(\phi, t) \equiv$ $V(\phi, t)$, then we obtain an effective QIF-NFM in variables $R$ and $V$,

$$
\begin{aligned}
\tau \frac{\partial R}{\partial t} & =\frac{\Delta}{\pi \tau}+2 R V, \\
\tau \frac{\partial V}{\partial t} & =V^{2}+\bar{\eta}-(\pi \tau R)^{2}+\tau S(\phi)+P(\phi, t) .
\end{aligned}
$$

In this case, the mean field reduces to

$$
S(\phi)=\frac{1}{2 \pi} \int_{-\pi}^{\pi}\left[J_{0}+2 \sum_{K=1}^{\infty} J_{K} \cos \left(K\left(\phi^{\prime}-\phi\right)\right)\right] R\left(\phi^{\prime}\right) d \phi^{\prime},
$$

with the new Fourier coefficients $J_{K}$, which are related to those in Eq. (1) as $J_{K}=J_{K}^{e}-J_{K}^{i}$, with $K=0,1, \ldots$, see Fig. 1(d). Solutions to Eq. (6) need not satisfy the condition $R^{e}=R^{i}$ and $V^{e}=V^{i}$, but the reduced system Eq. (7) captures the mechanism behind the oscillatory behavior observed in the model. Note that, in Figs. 2(a) and 2(b), we perturbed the SHS of the system [Eqs. (2) and (3)] using a current pulse to all the excitatory neurons. The resulting dynamics is only captured by the full system [Eqs. (6)] and not by the effective neural field [Eqs. (7)]. However, we next show that the existence of the spatial oscillatory modes observed in Fig. 2 is exclusively linked to the dynamics in the reduced manifold defined by Eqs. (7) and (8).

\section{B. SHS and their stability: Synchrony-induced modes of oscillation}

In the following we investigate the stability of the stationary SHS of the QIF-NFM against spatial perturbations. The detailed linear stability analysis of both the complete model [Eqs. (6)] and the reduced one [Eqs. (7)] are provided in Appendix B. 
In absence of external inputs, $P(\phi, t)=0$, the steady states of Eqs. (7)—and also of Eqs. (6)-satisfy $V_{*}(\phi)=$ $-\Delta /\left[2 \pi \tau R_{*}(\phi)\right]$, and

$$
R_{*}(\phi)=\Phi\left[\bar{\eta}+\tau S_{*}(\phi)\right],
$$

with $\Phi(x)=\sqrt{x+\sqrt{x^{2}+\Delta^{2}}} /(\sqrt{2} \pi \tau)$. In Eq. (9), the term $S_{*}(\phi)$ is the mean field [Eq. (8)] evaluated at $R_{*}(\phi)$. For SHS, the mean field [Eq. (8)] becomes spatially independent, $S_{*}(\phi)=S_{*}=J_{0} R_{*}$, and Eq. (9) becomes a quartic equation for the variable $R_{*}$. To further simplify the analysis, hereafter we consider parameter ranges where Eq. (9) has a single positive root. Accordingly, we consider a balanced kernel, $J_{0}=0$ so that Eq. (9) has $S_{*}=0$ and explicitly determines the value of the fixed point $R_{*}$.

The steady states of the SHS of Eq. (7) coincide with those of a single population of neurons [28]. However, the stability of the SHS of the QIF-NFM to inhomogeneous perturbations depends on the spatial character of the connectivity kernel Eq. (1). The linear stability analysis of the SHS gives a countably infinite set of eigenvalues associated to the stability of perturbations with wave number $K$ [34],

$\lambda_{K \pm}=-\frac{\Delta}{\pi \tau^{2} R_{*}} \pm 2 \pi R_{*} \sqrt{\frac{J_{K}}{2 \pi^{2} \tau R_{*}}-1}, \quad(K=0,1,2 \ldots)$.

This equation is the main result of this work, and explains the synchronization patterns shown in Fig. 2. Note that the eigenvalues Eq. (10) may be real or complex, indicating nonoscillatory or oscillatory dynamics of the evolution of perturbations of wave number $K$, respectively. In particular, perturbations of any given spatial mode $K$ are oscillatory if the condition $J_{K}<2 \pi^{2} \tau R_{*}$ is fulfilled. Notably, all complex eigenvalues have the same decay rate to the SHS, since $\operatorname{Re}\left(\lambda_{K \pm}\right)=-\Delta /\left(\pi \tau^{2} R_{*}\right)$ for all of them. Specifically, the decay rate is proportional to the degree of quenched heterogeneity $\Delta$. This reflects the fact that the decay in the oscillations is in fact a desynchronization mechanism due to the distribution of inputs that the cells receive.

Substituting Eq. (9) with $J_{0}=0$ into Eq. (10), it is straightforward to find the boundary

$$
J_{K}^{o}=\sqrt{2} \pi \sqrt{\bar{\eta}+\sqrt{\bar{\eta}^{2}+\Delta^{2}}},
$$

separating the parameter space into regions where standing waves of wave number $K$ are, or are not, observed. This boundary is depicted with a dotted line in the phase diagram Fig. 3, together with a schematic representation of the location of the eigenvalues $\lambda_{K \pm}$ in the complex plane (red crosses, see also Fig. 5(a).

A given oscillatory mode $K$ has an associated frequency $v_{K}=1 /(2 \pi)\left|\operatorname{Im}\left(\lambda_{K \pm}\right)\right|$, which differs from one another depending on the corresponding Fourier coefficients $J_{K}$ of the patterns of synaptic connectivity Eq. (1). Therefore, spatial perturbations of wave number $K$ produce standing waves of neural activity of frequency $v_{K}$. Locally excitatory coupling $J_{K}>0$ slows down these oscillations and eventually suppresses them, whereas locally inhibitory coefficients $J_{K}<0$ are able to generate arbitrarily fast oscillations (in particular, note that all modes with $J_{K}=0$ are oscillatory with frequency (a)

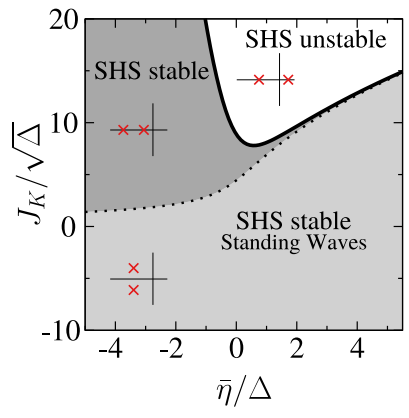

(b)

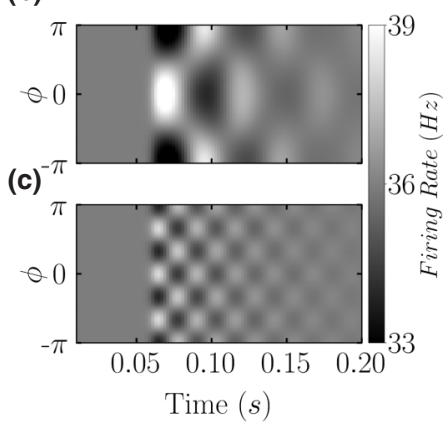

FIG. 3. (a) Phase diagram of Eqs. (7) (with $J_{0}=0$ ) showing the regions of stability of the SHS, determined by the eigenvalues Eq. (10). Spatial perturbations of wave number $K>0$ show oscillatory and nonoscillatory decay to the spatially homogeneous state in the light-shaded and dark-shaded regions of the diagram, respectively. The eigenvalues $\lambda_{K \pm}$ associated with the $K$ th mode are schematically represented in the complex plane (red crosses) for the three qualitatively different regions of the phase diagram. Right panels show the response of the Eqs. (7) with $J_{1}=10, J_{2}=7.5, J_{3}=-2.5$, and $J_{K}=0(K \neq 1,2,3), \bar{\eta}=4.5, \Delta=1$, and $\tau=20 \mathrm{~ms}$, to a perturbation of the (b) $K=1$ and (c) $K=3$ spatial modes. Both perturbations produce standing waves with frequency and decay rate described by Eqs. (10). In the white region, limited by the curve Eq. (11), these perturbations grow and lead to a bump state (BS) with $K$ bumps (see Fig. 4).

$v=R_{*}$, which coincides with the mean firing rate of the uncoupled neurons).

Indeed, in Fig. 2(d), a perturbation of wave number $K=3$ produced standing waves, since $J_{3}$ was negative. The frequency of these oscillations was fast compared to that of Fig. 2(c), where the exited mode was the first one $K=1$ and given that the $J_{1}$ was positive. However, note that in both cases the decay to the SHS is similar, as predicted by the eigenvalues Eq. (10). This indicates that the desynchronization process occurs faster when the diversity $\Delta$ of neurons is increased, and this process does not depend on the oscillation mode being excited. Finally, in Figs. 3(b) and 3(c) we show numerical simulations of the QIF-NFM [Eq. (7)] using the same parameters as those of Figs. 2 (c) and 2(d), and the agreement is good.

\section{Turing bifurcation and nonlinear stability of the SHS}

As $J_{K}$ is increased, the frequency $v_{K}$ of a given oscillatory mode decreases and eventually it ceases to oscillate. Further increases in $J_{K}$ may destabilize the homogeneous state, via a pattern-forming (Turing) bifurcation. This instability leads to states with spatially modulated firing rate, sometimes referred to as the bump states (BS). Substituting the fixed point (9) in Eq. (10), and imposing the condition of marginal stability $\lambda_{K+}=0$, we find the stability boundaries corresponding to a $K$-spatial mode,

$$
J_{K}^{T}=2 \pi \sqrt{\frac{2 \bar{\eta}^{2}+2 \Delta^{2}}{\bar{\eta}+\sqrt{\bar{\eta}^{2}+\Delta^{2}}}} .
$$



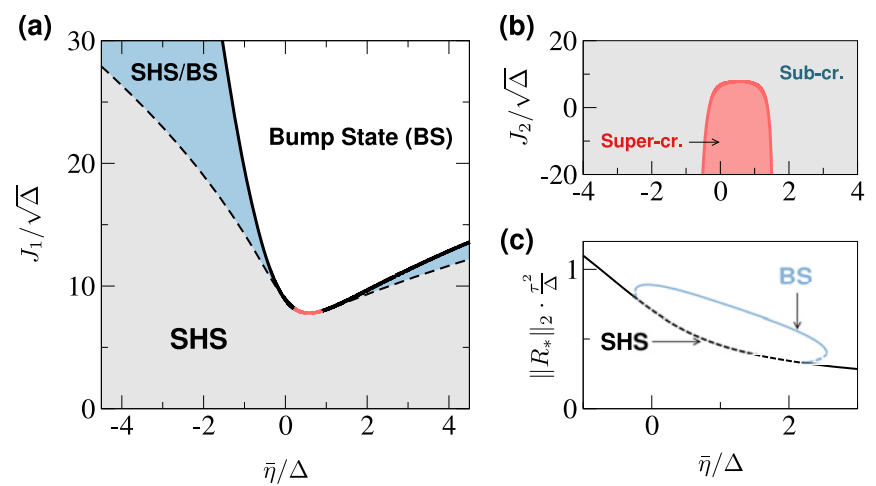

FIG. 4. (a) Phase diagram of the QIF-NFM [Eqs. (7)] with $J_{2}=7.5, J_{3}=-2.5, J_{K}=0$, for $K>3$, and $\Delta=1$. Solid line: supercritical [red (gray)] and subcritical (black) Turing bifurcation boundary Eq. (12)]. Dashed lines: saddle-node bifurcation of bumps (numerical). (b) Diagram—obtained using a weakly nonlinear analysis-showing the regions where the Turing bifurcation is supercritical or subcritical for $J_{1}=10, J_{3}=-2.5$, and $J_{K}=0$. (c) Bifurcation diagram (rescaled) $\left\|R_{*}\right\|_{2}=(2 \pi)^{-1} \int_{-\pi}^{\pi}\left|R_{*}(\phi)\right|^{2} d \phi$ vs. $\bar{\eta}$, for $J_{1}=10$. Solid/dashed black lines: stable/unstable SHS. Solid/dashed blue (light gray) lines: stable/unstable bump states (BS).

The Turing bifurcation boundary, Eq. (12), corresponds to the solid line in Figs. 3(a) and 4(a). Additionally, in Appendix C, we conducted a weakly nonlinear analysis and derived the small-amplitude equation [Eq. (C20)] corresponding to the bump solution bifurcating from the SHS. The amplitude equations determine if the Turing bifurcation is supercritical or if it is subcritical and bistability between SHS and bump states is expected to occur. The results of this analysis are summarized in Fig. 4(b).

In addition, we performed numerical simulations of the QIF-NFM (7) and indeed found coexistence of SHS and bump states in the blue-shaded regions limited by solid and dashed curves in Fig. 4(a). These lines meet at two codimension2 points (where the Turing bifurcation line changes color) that agree with the results of the weakly nonlinear analysis. Moreover, we computed numerically a bifurcation diagram of the NFM, using the spectral method developed in Ref. [35] and available with Ref. [36]. The results, presented in Fig. 4(c) confirm that the unstable BS bifurcates subcritically for the SHS. The unstable BS then meets a stable BS- - solid blue (light gray) line - at a fold bifurcation.

\section{Synchrony-induced transient oscillations in bump states}

To investigate whether the synchrony-induced oscillatory modes are also present in the stationary BS, we computed their spectrum. The gray points in Fig. 5(a) show the spectrum of the unstable Bump near the subcritical Turing bifurcation of wavelength $K=1$. Additionally, the red crosses in Fig. 5(a) are the eigenvalues of the SHS state Eq. (10). The profile of the unstable bump is only very weakly modulated, see Fig. 5(c), and hence the spectrum of the BS is very close to that of the SHS, given by the eigenvalues $\lambda_{K}$. All these eigenvalues are complex, except two real eigenvalues, which correspond to the $K=1$ mode. One of these eigenvalues is negative and the
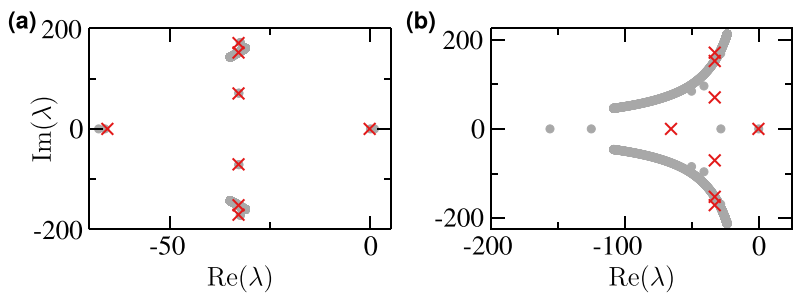

(c)
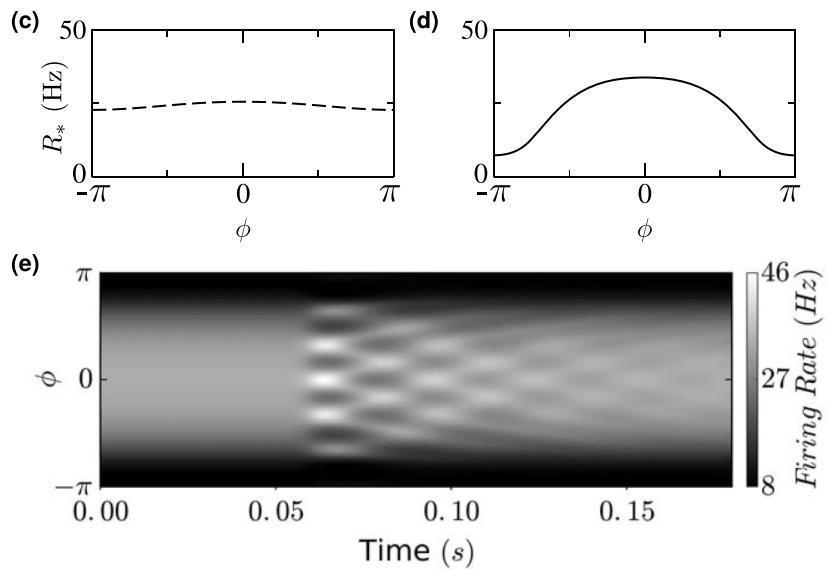

FIG. 5. Spectrum [(a) and (b)] and firing rate profiles [(d) and (c)] of an unstable [(a) and (c)] and stable [(b) and (d)] bump states of the QIF-NFM [Eqs. (7)]. In panels (a) and (b) the eigenvalues Eq. (10) are superimposed with red crosses. Panel (e) shows a numerical simulation of the BS of panel(d). At $t=0.05 \mathrm{~s}$, a perturbation of wave number $K=6$ is applied. Parameters are $J_{0}=0, J_{1}=10, J_{2}=7.5$, $J_{3}=-2.5, J_{K}=0$ for $K>3, \Delta=1, \tau=20 \mathrm{~ms}$. Panels (a) and (c): $\bar{\eta}=2.2120$; panels (b), (d), and (e): $\bar{\eta}=2.1828$.

other is very close to zero and positive, indicating that the SHS is unstable.

Additionally, it is important to note that in Fig. 5 we have taken $J_{K}=0$ for all $K$ except for $K=1,2,3$, and hence there is an infinite number of eigenvalues $\left(\lambda_{0}\right.$ and $\left.\lambda_{4,5}, \ldots\right)$ that are all complex and identical. In Fig. 5(a), the eigenvalues of the unstable BS seem to form a continuous band precisely around these infinitely degenerated eigenvalues and their complex conjugates. These continuous bands grow in size as one moves away from the Turing bifurcation, as it can be seen in the spectrum of the stable bump depicted in Fig. 5(b)-here red crosses also correspond to the eigenvalues of the SHS state Eq. (10). These results show that all the complex eigenvalues linked to the oscillatory modes of the SHS remain complex, suggesting that, in general, similar synchronizationinduced oscillations may be present in stationary, spatially inhomogeneous neural patterns.

Finally, to illustrate this, in Fig. 5(e) we performed a numerical simulation of the QIF-NFM Eqs. (7) and perturbed the BS shown in Fig. 5(d) with a spatially inhomogeneous perturbation corresponding to the mode $(K=6)$. The perturbation decays to the BS showing a pattern that resembles that of Fig. 2. However, here the regions of the ring with the maximum values of $R_{*}$-around $\phi=0$, in Figs. 2(d) and 2(e)-oscillate at high frequencies and these oscillations slow down as $\phi \rightarrow \pm \pi$. The spectrum of the stable BS Fig. 5(b) also indicates that the decay of the fast oscillations (located at the 
central part of the bump, $\phi=0$ ) is slow compared to that of the slow oscillations.

\section{CONCLUSIONS}

We have reported the existence of a class of oscillatory modes in spatially distributed networks of heterogeneous spiking neurons. These modes of oscillation reflect the transient episodes of spike synchronization among the neurons and are not captured by traditional NFMs.

To investigate these oscillation modes we derived a novel NFM for QIF neurons [Eqs. (6)]. Alternately, and invoking the Ott-Antonsen theory for populations of pulse-coupled theta neurons [37,38], Laing recently derived a NFM [39] that is equivalent to the effective QIF-NFM [Eqs. (7)]. In Laing's work-like in other recent related papers on pulse-coupled oscillators [40-45]-the resulting low-dimensional description is in terms of the complex Kuramoto order parameter. In contrast, the mean-field description adopted here (in terms of mean firing rates and membrane potentials) greatly simplifies the analysis, allowing us to analytically investigate the linear and nonlinear stability of the spatially homogeneous states of the QIF-NFM.

This analysis reveals two important features: (i) The frequency of each oscillation mode only depends on the corresponding Fourier coefficient of the synaptic pattern of connectivity, and (ii) the decay rate is exactly the same for all modes and is due to a desynchronization mechanism which depends on the degree of quenched heterogeneity. We also numerically investigated networks of identical QIF neurons subject to noise and found similar results (not shown). In this case, the desynchronization reflects an underlying phase diffusion proportional to the noise strength. Finally, we investigated the existence and stability of bump states, which bifurcate from the spatially homogeneous states via Turing bifurcations. The spectrum of such bump states has a continuous part off the real axis, indicating that similar synchronization-induced oscillatory modes also operate in neural bump states.

Interesting directions of further study are the analysis of the QIF-NFM [Eq. (6)] considering different membrane time constants $\tau$ (or different main currents $\bar{\eta}$ ) for excitatory and inhibitory neurons. As proved recently [46], NFMs with timescale separation display a rich variety of robust spatiotemporal patterns, which may also be supported by our model. Also, recent work has been done to extend the local firing rate equations derived in Ref. [28] to include fixed synaptic delays [47] and synaptic kinetics [48,49]—see also Refs. [43,44]. This work shows that time delays due to synaptic processing generally lead to the emergence of self-sustained oscillations due to collective synchronization. Extending the QIF-NFM [Eq. (6)] to account for the synaptic time delays caused by synaptic processing may lead to spatiotemporal phenomena not previously observed in traditional NFMs.

\section{ACKNOWLEDGMENTS}

J.M.E.-A. and E.M acknowledge support by the European Union's Horizon 2020 research and innovation programme under the Marie Skłodowska-Curie Grant No. 642563. J.M.E.-
A. and E.M. acknowledge the projects grants from the Spanish Ministry of Economics and Competitiveness, Grants No. PSI2016-75688-P and No. PCIN-2015-127. A.R. acknowledges a project grant from the Spanish ministry of Economics and Competitiveness, Grant No. BFU2012-33413. A.R. has been partially funded by the CERCA progam of the Generalitat de Catalunya. D.A. was partially supported by the EPSRC Grant No. EP/P510993/1 (United Kingdom).

\section{APPENDIX A: DERIVATION OF THE QIF NEURAL FIELD MODEL (QIF-NFM)}

Our derivation closely follows that of Ref. [28], but it needs to be extended to include the spatial dimension. Similar extensions from a single population of phase oscillators to a one-dimensional, spatially distributed network with nonlocal coupling have been done in Refs. [39,50-56].

Considering the thermodynamic limit $N \rightarrow \infty$, we can drop the indexes in Eqs. (2) and (3) and define the density function $\rho^{e, i}\left(v^{e, i} \mid \eta^{e, i}, t, \phi\right)$ such that $\rho^{e, i}\left(v^{e, i} \mid \eta^{e, i}, t, \phi\right) d v^{e, i} d \eta^{e, i} d \phi$ describes the fraction of neurons located between $\phi$ and $\phi+d \phi$, with membrane potentials between $v^{e, i}$ and $v^{e, i}+$ $d v^{e, i}$ and parameters between $\eta^{e, i}$ and $\eta^{e, i}+d \eta^{e, i}$ at time $t$. Accordingly, parameter $\eta^{e, i}$ becomes now a continuous random variable with probability density function $g\left(\eta^{e, i}\right)$. For the sake of simplicity, we assume identical distributions for both excitatory and inhibitory populations $g\left(\eta^{e, i}\right)=g(\eta)$. The total voltage density at location $\phi$ and time $t$ is given by $\int_{-\infty}^{\infty} \rho^{e, i}\left(v^{e, i} \mid \eta, t, \phi\right) g(\eta) d \eta$.

Conservation of the number of neurons at each $\phi$ value is described by the continuity equation

$$
\partial_{t} \rho^{e, i}=-\partial_{v}\left[\left(\left(v^{e, i}\right)^{2}+\eta+\tau S(\phi, t)+P^{e, i}(\phi, t)\right) \rho^{e, i}\right],
$$

where we have explicitly included the velocity given by Eqs. (2) and (3) and $S(\phi, t)=S^{e}(\phi, t)+S^{i}(\phi, t)$ represents the total synaptic activity. Next we invoke the Ott-Antonsen theory [29] by means of the Lorentzian ansatz (LA) [28],

$$
\rho^{e, i}\left(v^{e, i} \mid \eta, t, \phi\right)=\frac{1}{\pi} \frac{x^{e, i}(\phi, \eta, t)}{\left[v^{e, i}-y^{e, i}(\phi, \eta, t)\right]^{2}+x^{e, i}(\phi, \eta, t)^{2}},
$$

which solves the continuity equation. The width $x^{e, i}(\phi, \eta, t)$ of the LA is related to the firing rate $R^{e, i}$ of the neural populations. Indeed, for each $\eta$ value at time $t, R^{e, i}(\phi, \eta, t)$ can be evaluated noting that neurons fire at a rate given by the probability flux at infinity: $R^{e, i}(\phi, \eta, t)=\rho^{e, i}\left(v^{e, i} \rightarrow\right.$ $\infty \mid \eta, t, \phi) \dot{v}^{e, i}\left(v^{e, i} \rightarrow \infty \mid \eta, t, \phi\right)$. The limit $v^{e, i} \rightarrow \infty$ on the right-hand side of this equation can be evaluated within the LA and gives $x^{e, i}(\phi, \eta, t)=\pi \tau R^{e, i}(\phi, \eta, t)$. The total firing rate at a particular location $\phi$ of the ring is then

$$
R^{e, i}(\phi, t)=\frac{1}{\tau \pi} \int_{-\infty}^{\infty} x^{e, i}(\phi, \eta, t) g(\eta) d \eta .
$$

Additionally, the quantity $y^{e, i}(\eta, t)$ is, for each value of $\eta$, the mean of the membrane potential $y^{e, i}(\phi, \eta, t)=$ P.V. $\int_{-\infty}^{\infty} \rho^{e, i}\left(v^{e, i} \mid \eta, t, \phi\right) v^{e, i} d v^{e, i}$. Therefore, this variable is related to the mean membrane potential of the neuronal 
population at $\phi$ by

$$
V^{e, i}(\phi, t)=\int_{-\infty}^{\infty} y^{e, i}(\phi, \eta, t) g(\eta) d \eta
$$

Substituting the LA (A1) into the continuity equation, we find that, for each value of $\eta$, the variables $x^{e, i}(\phi)$ and $y^{e, i}(\phi)$ must obey two coupled equations which can be written in complex form as

$\tau \partial_{t} w^{e, i}(\phi, \eta, t)=i\left[\eta+\tau S(\phi, t)-\left(w^{e, i}\right)^{2}(\phi, \eta, t)+P^{e, i}(\phi, t)\right]$,

where $w^{e, i}(\phi, \eta, t) \equiv x^{e, i}(\phi, \eta, t)+i y^{e, i}(\phi, \eta, t)$. If $\eta$ are distributed according to a Lorentzian distribution Eq. (5), the integrals in (A2) and (A3) can then be evaluated closing the integral contour in the complex $\eta$ plane and using the Cauchy residue theorem. Then the firing rate and mean membrane potential depend only on the value of $w^{e, i}$ at the pole of $g(\eta)$ in the lower half $\eta$ plane: $\pi \tau R^{e, i}(\phi, t)+i V^{e, i}(\phi, t)=$ $w^{e, i}(\phi, \bar{\eta}-i \Delta, t)$, and, as a result, (A4) must be evaluated only at $\eta=\bar{\eta}-i \Delta$ to obtain the neural field equations [Eq. (6)] [57].

These equations can be nondimensionalized by rescaling variables and time as (note the difference between $v_{j}^{e, i}$, the membrane potential of a single neuron $j$, and the mean membrane potential $v^{e, i}$ ):

$$
R^{e, i}=\frac{\sqrt{\Delta}}{\tau} r^{e, i}, V^{e, i}=\sqrt{\Delta} v^{e, i}, t=\frac{\tau}{\sqrt{\Delta}} \tilde{t},
$$

and parameters as

$$
J_{K}^{e, i}=\sqrt{\Delta} j_{K}^{e, i}, \bar{\eta}=\Delta \tilde{\eta}, P^{e, i}(\phi, t)=\Delta \tilde{P}^{e, i}(\phi, \tilde{t}) .
$$

The resulting dimensionless NFM is then

$$
\begin{aligned}
& \dot{r}^{e, i}=\frac{1}{\pi}+2 v^{e, i} r^{e, i}, \\
& \dot{v}^{e, i}=\left(v^{e, i}\right)^{2}+\tilde{\eta}-\pi^{2}\left(r^{e, i}\right)^{2}+s(\phi, \tilde{t})+\tilde{P}^{e, i}(\phi, \tilde{t}),
\end{aligned}
$$

where the overdot represents partial derivation with respect the nondimensional time $\tilde{t}$ and the mean field is

$$
\begin{aligned}
s(\phi, \tilde{t})= & \frac{1}{\pi} \int_{-\pi}^{\pi}\left[\frac{j_{0}^{e}}{2}+\sum_{K=1}^{\infty} j_{K}^{e} \cos \left(K\left(\phi^{\prime}-\phi\right)\right)\right] r^{e}\left(\phi^{\prime}, \tilde{t}\right) d \phi^{\prime} \\
& -\frac{1}{\pi} \int_{-\pi}^{\pi}\left[\frac{j_{0}^{i}}{2}+\sum_{K=1}^{\infty} j_{K}^{i} \cos \left(K\left(\phi^{\prime}-\phi\right)\right)\right] r^{i}\left(\phi^{\prime}, \tilde{t}\right) d \phi^{\prime} .
\end{aligned}
$$

\section{Effective NFM model}

Considering $\tilde{P}^{e, i}(\phi, \tilde{t})=\tilde{P}(\phi, \tilde{t})$ in Eqs. (A7), the system

$$
\begin{aligned}
& \dot{r}=\frac{1}{\pi}+2 v r, \\
& \dot{v}=v^{2}+\tilde{\eta}-\pi^{2} r^{2}+s(\phi, \tilde{t})+\tilde{P}(\phi, \tilde{t}),
\end{aligned}
$$

with the mean field

$$
s(\phi, t)=\frac{1}{\pi} \int_{-\pi}^{\pi}\left[\frac{j_{0}}{2}+\sum_{K=1}^{\infty} j_{K} \cos \left(K\left(\phi^{\prime}-\phi\right)\right)\right] r\left(\phi^{\prime}, t\right) d \phi^{\prime}
$$

and

$$
j_{K}=j_{K}^{e}-j_{K}^{i}
$$

has identical symmetric solutions as the original Eqs. (A7), i.e.,

$$
r^{e}(t)=r^{i}(t)=r(t), \quad v^{e}(t)=v^{i}(t)=v(t) .
$$

\section{APPENDIX B: LINEAR STABILITY ANALYSIS OF THE SPATIALLY HOMOGENEOUS STATE}

\section{Linear stability of effective QIF-NFM Eq. (7)}

The homogeneous steady state is given by the solution of Eq. (9) when $R_{*}(\phi)=R_{*}$. This is equivalent to $S_{*}(\phi)=S_{*}=$ $J_{0} R_{*}$ that in dimensionless form is

$$
\pi^{2} r_{*}^{4}-j_{0} r_{*}^{3}-\tilde{\eta} r_{*}^{2}-\frac{1}{4 \pi^{2}}=0
$$

This equation is greatly simplified assuming $j_{0}=0$ and gives

$$
r_{*}=\frac{1}{\pi \sqrt{2}} \sqrt{\tilde{\eta}+\sqrt{\tilde{\eta}^{2}+1}} .
$$

The stability of homogeneous steady-state solutions can be analyzed studying the evolution of the small (even) perturbations $(\epsilon \ll 1)$ of the SHS

$$
\begin{aligned}
& r(\phi, t)=r_{*}+\epsilon \sum_{K=0}^{\infty} a_{K}(t) \cos (K \phi), \\
& v(\phi, t)=v_{*}+\epsilon \sum_{K=0}^{\infty} b_{K}(t) \cos (K \phi) .
\end{aligned}
$$

Substituting (B3) into the mean field (A10), we obtain a perturbed mean field around $s_{*}(\phi)$

$$
s(\phi, t)=s_{*}(\phi)+\epsilon \sum_{K=0}^{\infty} j_{K} a_{K}(t) \cos (K \phi) .
$$

Linearizing Eqs. (A9) around the fixed point $\left(r_{*}, v_{*}\right)$, gives

$$
\begin{aligned}
\sum_{K=0}^{\infty} \mu_{K} a_{K} \cos (K \phi)= & 2 \sum_{K=0}^{\infty}\left[r_{*}(\phi) b_{K}+v_{*}(\phi) a_{K}\right] \cos (K \phi), \\
\sum_{K=0}^{\infty} \mu_{K} b_{K} \cos (K \phi)= & \sum_{K=0}^{\infty}\left[2 v_{*}(\phi) b_{K}+\left(j_{K}-2 \pi^{2} r_{*}(\phi)\right) a_{K}\right] \\
& \times \cos (K \phi),
\end{aligned}
$$

where we have used the ansatz $a_{K}(t)=a_{K} e^{\mu_{K} t}$ and $b_{K}(t)=$ $b_{K} e^{\mu_{K} t}$, where $\mu_{K}$ represents the dimensionless eigenvalue of the $K$ th mode. For SHS states, $\left(r_{*}(\phi), v_{*}(\phi)\right)=\left(r_{*}, v_{*}\right)$, the modes in Eqs. (B5) decouple and, for a given mode $K$, we find the linear system

$$
\mu_{K}\left(\begin{array}{l}
a_{K} \\
b_{K}
\end{array}\right)=L_{*}\left(\begin{array}{l}
a_{K} \\
b_{K}
\end{array}\right)
$$


with

$$
L_{*}=\left(\begin{array}{cc}
2 v_{*} & 2 r_{*} \\
j_{K}-2 \pi^{2} r_{*} & 2 v_{*}
\end{array}\right) .
$$

Equation (B6) has a general solution:

$$
\left(\begin{array}{l}
a_{K}(\tilde{t}) \\
b_{K}(\tilde{t})
\end{array}\right)=A_{+} \mathbf{u}_{+} e^{\mu_{K+} \tilde{t}}+A_{-} \mathbf{u}_{-} e^{\mu_{K-} \tilde{t}},
$$

where $A_{ \pm}$are arbitrary constants. The eigenvalues $\mu_{K \pm}$ are given by

$$
\mu_{K \pm}=-\frac{1}{\pi r_{*}} \pm 2 \pi r_{*} \sqrt{\frac{j_{K}}{2 \pi^{2} r_{*}}-1},
$$

with eigenvectors

$$
\mathbf{u}_{ \pm}=\left(\frac{ \pm 1}{\sqrt{\frac{j_{K}}{2 r_{*}}-\pi^{2}}}\right) .
$$

In terms of the dimensional variables and parameters (A5) and (A6), the eigenvalues (B9) are $\lambda_{k} t=\mu_{k} \tilde{t}$, and thus $\lambda_{k}=$ $\sqrt{\Delta} \mu_{k} / \tau$, which gives the eigenvalues Eq. (10) in the main text.

\section{Linear stability of the full QIF-NFM}

For the full QIF-NFM [Eq. (6)], the perturbation around the SHS state has the form

$$
\begin{aligned}
& r^{e, i}(\phi, t)=r_{*}+\epsilon \sum_{K=0}^{\infty} a_{K}^{e, i}(t) \cos (K \phi), \\
& v^{e, i}(\phi, t)=v_{*}+\epsilon \sum_{K=0}^{\infty} b_{K}^{e, i}(t) \cos (K \phi) .
\end{aligned}
$$

In this case, the linear stability of the SHS state with respect to perturbations of the $K$-spatial mode is determined by the characteristic equation

$$
\begin{aligned}
\lambda_{K}\left(\begin{array}{l}
a_{K}^{e} \\
b_{K}^{e} \\
a_{K}^{i} \\
b_{K}^{i}
\end{array}\right)= & \left(\begin{array}{cccc}
2 v_{*} & 2 r_{*} & 0 & 0 \\
j_{K}^{e}-2 \pi^{2} r_{*} & 2 v_{*} & -j_{K}^{i} & 0 \\
0 & 0 & 2 v_{*} & 2 r_{*} \\
j_{K}^{e} & 0 & -j_{K}^{i}-2 \pi^{2} r_{*} & 2 v_{*}
\end{array}\right) \\
& \times\left(\begin{array}{l}
a_{K}^{e} \\
b_{K}^{e} \\
a_{K}^{i} \\
b_{K}^{i}
\end{array}\right) .
\end{aligned}
$$

For each $K$ mode, the linearized system has a general solution,

$$
\begin{aligned}
\left(\begin{array}{c}
a_{K}^{e}(\tilde{t}) \\
b_{K}^{e}(\tilde{t}) \\
a_{K}^{i}(\tilde{t}) \\
b_{K}^{i}(\tilde{t})
\end{array}\right)= & A_{+} \mathbf{u}_{K+} e^{\mu_{K+} \tilde{t}}+A_{-} \mathbf{u}_{K-} e^{\mu_{K-} \tilde{t}} \\
& +B_{+} \mathbf{u}_{K \perp} e^{\mu_{\perp} \tilde{t}}+B_{-} \overline{\mathbf{u}}_{K \perp} e^{\bar{\mu}_{\perp} \tilde{t}}
\end{aligned}
$$

where $A_{ \pm}$and $B_{ \pm}$are arbitrary constants. The eigenvectors

$$
\mathbf{u}_{K \pm}=\left(\begin{array}{c} 
\pm 1 \\
\sqrt{\frac{j_{K}^{e}-j_{K}^{i}}{2 r_{*}}-\pi^{2}} \\
\pm 1 \\
\sqrt{\frac{j_{K}^{e}-j_{K}^{i}}{2 r_{*}}-\pi^{2}}
\end{array}\right)
$$

have eigenvalues

$$
\mu_{K \pm}=-\frac{1}{\pi r_{*}} \pm 2 \pi r_{*} \sqrt{\frac{j_{K}^{e}-j_{K}^{i}}{2 \pi^{2} r_{*}}-1}
$$

These eigenvalues coincide with those of the reduced system (B9) and are associated with the standing waves shown in Fig. 2. Additionally, the eigenvector

$$
\mathbf{u}_{K \perp}=\left(\begin{array}{c}
i j_{K}^{i} \\
\pi j_{K}^{i} \\
i j_{K}^{e} \\
\pi j_{K}^{e}
\end{array}\right),
$$

and its complex conjugate $\overline{\mathbf{u}}_{K \perp}$, with associated eigenvalue

$$
\mu_{\perp}=-\frac{1}{\pi r_{*}}+i 2 \pi r_{*} .
$$

and its complex conjugate $\bar{\mu}_{\perp}$, correspond to modes of oscillation of the uncoupled system. Indeed, note that the eigenvalues (B16) are independent of the connectivity and correspond to oscillatory modes which are already present in a single population of uncoupled neurons - note that eigenvalues (B14) reduce to (B16) for all the modes with $j_{K}=j_{K}^{e}-j_{K}^{i}=0$.

\section{APPENDIX C: SMALL-AMPLITUDE EQUATION NEAR THE SPATIALLY HOMOGENEOUS STATE}

\section{Critical eigenvectors}

Right at the bifurcation, the only undamped mode is the critical one given by $\mathbf{u}_{+}$in (B15) that reduces to the critical eigenmode:

$$
\mathbf{u}_{c}=\left(\begin{array}{c}
r_{*} \\
-v_{*}
\end{array}\right) .
$$

At criticality, the critical eigenmode of $L_{*}$ satisfies

$$
L_{* c} \mathbf{u}_{c}=0,
$$

where $L_{* c}$ corresponds to the operator (B7) evaluated at $j_{K}=$ $j_{K c}$. The left critical eigenvector of the operator $L_{* c}$ is then defined as

$$
\mathbf{u}_{c}^{\dagger} L_{* c}=0
$$

which gives

$$
\mathbf{u}_{c}^{\dagger}=\pi\left(\begin{array}{c}
-v_{*} \\
r_{*}
\end{array}\right)^{T},
$$

where the constant has been taken to normalize the eigenvectors, so that they satisfy $\mathbf{u}_{c}^{\dagger} \mathbf{u}_{c}=1$. 


\section{Amplitude equation}

Except for initial transients, the amplitude of the bifurcating solution at criticality is expected to contain only the component $\mathbf{u}_{+c}$. In the following we derive a small-amplitude equation for the bump solutions using multiple-scale analysis, see, e.g., Ref. [58]. First, let the solution of Eqs. (A9) be written as the perturbation expansion

$$
\left(\begin{array}{l}
r(\phi, \tilde{t}) \\
v(\phi, \tilde{t})
\end{array}\right)=\left(\begin{array}{l}
r_{*} \\
v_{*}
\end{array}\right)+\epsilon\left(\begin{array}{l}
r_{\epsilon}(\phi, \tilde{t}, \tilde{T}) \\
v_{\epsilon}(\phi, \tilde{t}, \tilde{T})
\end{array}\right)+\epsilon^{2}\left(\begin{array}{l}
r_{\epsilon \epsilon}(\phi, \tilde{t}, \tilde{T}) \\
v_{\epsilon \epsilon}(\phi, \tilde{t}, \tilde{T})
\end{array}\right)+\ldots
$$

where $\left(r_{*}, v_{*}\right)$ is the state SHS given by the solutions of (B1) and $\epsilon \ll 0$ is a small parameter, which measures the distance from the Turing bifurcation. In addition, we define a long time scale, $\tilde{T}=\epsilon^{2} \tilde{t}$, that is considered to be independent of $\tilde{t}$. Accordingly, the differential operator in Eqs. (A9) may be replaced by

$$
\partial_{\tilde{t}} \rightarrow \partial_{\tilde{t}}+\epsilon^{2} \partial_{\tilde{T}}
$$

Since the asymptotic expansion is going to be performed in the vicinity of a stationary bifurcation, we set $\partial_{\tilde{t}}=0$ so that the only temporal variations occur with the slow time scale $\tilde{T}$.

Additionally, in our analysis we use the parameter $j_{1}$ as the bifurcation parameter, and we write it as

$$
j_{1}=j_{1}^{T}+\epsilon^{2} \delta j_{1},
$$

where $j_{1}^{T}$ is the critical value of $j_{1}$ at which the Turing bifurcation occurs, given by Eq. (11), with $K=1$. Accordingly, the (nondimensionalized) connectivity footprint (1) is

$$
j(\phi)=j_{c}(\phi)+2 \epsilon^{2} \delta j_{1} \cos \phi,
$$

with

$$
j_{c}(\phi)=j_{0}+2 j_{1}^{T} \cos \phi+2 \sum_{K=2}^{\infty} j_{K} \cos (K \phi),
$$

where $j_{K}<j_{K c}$ for $K \neq 1$. To simplify the notation, we hereafter omit to explicitly write the dependence of $r_{\epsilon, \epsilon \epsilon, \ldots}$ and $v_{\epsilon, \epsilon \epsilon, \ldots}$ on the variables $\tilde{t}, T$, and $\phi$. Substituting (C3) and (C5) into the mean field (A10):

$$
\begin{aligned}
s(\phi)= & \frac{1}{2 \pi} \int_{-\pi}^{\pi}\left(r_{*}+\epsilon r_{\epsilon}+\epsilon^{2} r_{\epsilon \epsilon}+\ldots\right) j_{c}\left(\phi-\phi^{\prime}\right) d \phi^{\prime} \\
& +\epsilon^{2} \frac{1}{\pi} \int_{-\pi}^{\pi}\left(r_{*}+\epsilon r_{\epsilon}+\epsilon^{2} r_{\epsilon \epsilon}+\ldots\right) \delta j_{1} \cos \left(\phi-\phi^{\prime}\right) d \phi^{\prime} \\
\equiv & \left\langle r_{*}+\epsilon r_{\epsilon}+\epsilon^{2} r_{\epsilon \epsilon}+\ldots\right\rangle_{c}+2 \epsilon^{2}\left\langle r_{*}+\epsilon r_{\epsilon}+\epsilon^{2} r_{\epsilon \epsilon}+\ldots\right\rangle, \\
= & r_{*} j_{0}+\epsilon\left\langle r_{\epsilon}\right\rangle_{c}+\epsilon^{2}\left\langle r_{\epsilon \epsilon}\right\rangle_{c}+\epsilon^{3}\left(\left\langle r_{\epsilon \epsilon \epsilon}\right\rangle_{c}+2\left\langle r_{\epsilon}\right\rangle\right)+\ldots
\end{aligned}
$$

Plugging expansions (C3) and (C5) into the NFM Eqs. (A9), we obtain

$$
\begin{aligned}
& \epsilon^{2} \partial_{\tilde{T}}\left(\epsilon r_{\epsilon}+\epsilon^{2} r_{\epsilon \epsilon}+\ldots\right) \\
& =\quad \epsilon\left(2 v_{*} r_{\epsilon}+2 r_{*} v_{\epsilon}\right)+\epsilon^{2}\left(2 v_{*} r_{\epsilon \epsilon}+2 r_{\epsilon} v_{\epsilon}+2 r_{*} v_{\epsilon \epsilon}\right) \\
& \quad+\epsilon^{3}\left(2 v_{\epsilon} r_{\epsilon \epsilon}+2 r_{\epsilon} v_{\epsilon \epsilon}\right)+\ldots \\
& \epsilon^{2} \partial_{\tilde{T}}\left(\epsilon v_{\epsilon}+\epsilon^{2} v_{\epsilon \epsilon}+\ldots\right)
\end{aligned}
$$

$$
\begin{aligned}
= & \epsilon\left(2 v_{*} v_{\epsilon}-2 \pi^{2} r_{*} r_{\epsilon}+\left\langle r_{\epsilon}\right\rangle_{c}\right)+\epsilon^{2}\left(v_{\epsilon}^{2}-\pi^{2} r_{\epsilon}^{2}+2 v_{*} v_{\epsilon \epsilon}\right. \\
& \left.-2 \pi^{2} r_{*} r_{\epsilon \epsilon}+\left\langle r_{\epsilon \epsilon}\right\rangle_{c}\right) \\
& +\epsilon^{3}\left(2 v_{\epsilon} v_{\epsilon \epsilon}-2 \pi^{2} r_{\epsilon} r_{\epsilon \epsilon}+\left\langle r_{\epsilon \epsilon \epsilon}\right\rangle_{c}+2\left\langle r_{\epsilon}\right\rangle\right)+\ldots
\end{aligned}
$$

These equations can be written in a more compact form as

$$
\begin{gathered}
-\left(L_{c}+\epsilon^{2} L_{\epsilon \epsilon}\right)\left[\epsilon\left(\begin{array}{l}
r_{\epsilon} \\
v_{\epsilon}
\end{array}\right)+\epsilon^{2}\left(\begin{array}{l}
r_{\epsilon \epsilon} \\
v_{\epsilon \epsilon}
\end{array}\right)+\ldots\right] \\
=\epsilon^{2} N_{\epsilon \epsilon}+\epsilon^{3} N_{\epsilon \epsilon \epsilon}+\ldots,
\end{gathered}
$$

defining the linear and nonlinear operators

$$
\begin{aligned}
L_{c} & =\left(\begin{array}{cc}
2 v_{*} & 2 r_{*} \\
\langle\cdot\rangle_{c}-2 \pi^{2} r_{*} & 2 v_{*}
\end{array}\right), \\
L_{\epsilon \epsilon} & =\left(\begin{array}{cc}
-\partial_{\tilde{T}} & 0 \\
2\langle\cdot\rangle & -\partial_{\tilde{T}}
\end{array}\right), \\
N_{\epsilon \epsilon} & =\left(\begin{array}{c}
2 r_{\epsilon} v_{\epsilon} \\
v_{\epsilon}^{2}-\pi^{2} r_{\epsilon}^{2}
\end{array}\right), \\
N_{\epsilon \epsilon \epsilon} & =\left(\begin{array}{c}
2 r_{\epsilon} v_{\epsilon \epsilon}+2 r_{\epsilon \epsilon} v_{\epsilon} \\
2 v_{\epsilon} v_{\epsilon \epsilon}-2 \pi^{2} r_{\epsilon} r_{\epsilon \epsilon}
\end{array}\right),
\end{aligned}
$$

Next we collect terms by order in $\epsilon$. At first order we recover the linear problem (B6) at the Turing bifurcation:

$$
\left(\begin{array}{cc}
2 v_{*} & 2 r_{*} \\
j_{1}^{T}-2 \pi^{2} r_{*} & 2 v_{*}
\end{array}\right)\left(\begin{array}{l}
r_{\epsilon} \\
v_{\epsilon}
\end{array}\right)=\left(\begin{array}{l}
0 \\
0
\end{array}\right) .
$$

Recalling that $j_{1}^{T}$ is given by Eq. (C4), we find the neutral solution:

$$
\left(\begin{array}{l}
r_{\epsilon} \\
v_{\epsilon}
\end{array}\right)=A \mathbf{u}_{c} \cos \phi
$$

where $A$ is the small amplitude with slow time dependence that we aim to determine and $\mathbf{u}_{c}$ is the critical eigenmode given by Eq. (C1). Substituting the solution (C10) into the nonlinear forcing terms $N_{\epsilon \epsilon}$ we find

$$
N_{\epsilon \epsilon}=\frac{A^{2}}{2}\left(\begin{array}{c}
\pi^{-1} \\
v_{*}^{2}-\pi^{2} r_{*}^{2}
\end{array}\right)[1+\cos (2 \phi)]
$$

which implies that, at second order, the solution must necessarily contain homogeneous and second spatial components

$$
\left(\begin{array}{c}
r_{\epsilon \epsilon} \\
v_{\epsilon \epsilon}
\end{array}\right)=\left(\begin{array}{c}
r_{\epsilon \epsilon 0} \\
v_{\epsilon \epsilon 0}
\end{array}\right)+\left(\begin{array}{c}
r_{\epsilon \epsilon 2} \\
v_{\epsilon \epsilon 2}
\end{array}\right) \cos (2 \phi) .
$$

Equating the homogeneous, second-order terms of Eq. (C9), we find

$$
-\left(\begin{array}{cc}
2 v_{*} & 2 r_{*} \\
j_{0}-2 \pi^{2} r_{*} & 2 v_{*}
\end{array}\right)\left(\begin{array}{l}
r_{\epsilon \epsilon 0} \\
v_{\epsilon \in 0}
\end{array}\right)=\frac{A^{2}}{2}\left(\begin{array}{c}
\pi^{-1} \\
v_{*}^{2}-\pi^{2} r_{*}^{2}
\end{array}\right),
$$

and left-multiplying this equation by $L_{c}^{-1}$ and using Eq. (12), we find

$$
\left(\begin{array}{c}
r_{\epsilon \epsilon 0} \\
v_{\epsilon \epsilon 0}
\end{array}\right)=\frac{A^{2}}{4 r_{*}\left(j_{1}^{T}-j_{0}\right)}\left(\begin{array}{cc}
2 v_{*} & -2 r_{*} \\
2 \pi^{2} r_{*}-j_{0} & 2 v_{*}
\end{array}\right)\left(\begin{array}{c}
\pi^{-1} \\
v_{*}^{2}-\pi^{2} r_{*}^{2}
\end{array}\right),
$$


which gives the coefficients

$$
\begin{gathered}
r_{\epsilon \in 0}=\frac{3 v_{*}^{2}-\pi^{2} r_{*}^{2}}{2\left(j_{1}^{T}-j_{0}\right)} A^{2}, \\
v_{\epsilon \in 0}=\frac{2 \pi v_{*}^{4}-v_{*} j_{0}-3 \pi / 2}{2\left(j_{1}^{T}-j_{0}\right)} A^{2} .
\end{gathered}
$$

Proceeding similarly, we find the coefficients corresponding to the second spatial Fourier modes:

$$
\begin{gathered}
r_{\epsilon \in 2}=\frac{3 v_{*}^{2}-\pi^{2} r_{*}^{2}}{2\left(j_{1}^{T}-j_{2}\right)} A^{2}, \\
v_{\epsilon \in 2}=\frac{2 \pi v_{*}^{4}-v_{*} j_{2}-3 \pi / 2}{2\left(j_{1}^{T}-j_{2}\right)} A^{2} .
\end{gathered}
$$

Collecting the third-order terms of equation (C9), we obtain the identity

$$
-L_{c}\left(\begin{array}{c}
r_{\epsilon \epsilon \epsilon} \\
v_{\epsilon \epsilon \epsilon}
\end{array}\right)-L_{\epsilon \epsilon}\left(\begin{array}{l}
r_{\epsilon} \\
v_{\epsilon}
\end{array}\right)=N_{\epsilon \epsilon \epsilon},
$$

To obtain the desired amplitude equation, we shall leftmultiply Eq. (C15) by the left null-eigenvector (C2) and project it into the first spatial Fourier mode. The first term on the right-hand side of Eq. (C15) vanishes since $\mathbf{u}_{c}^{\dagger} L_{c}=0$. The second term is

$$
L_{\epsilon \epsilon}\left(\begin{array}{l}
r_{\epsilon} \\
v_{\epsilon}
\end{array}\right)=\left(\begin{array}{c}
-r_{*} \partial_{\tilde{T}} A \\
v_{*} \partial_{\tilde{T}} A+\delta j_{1} r_{*} A
\end{array}\right) \cos \phi .
$$

Finally, the nonlinear forcing term at the left-hand side of Eq. $(\mathrm{C} 15)$ is

$$
\begin{aligned}
N_{\epsilon \epsilon \epsilon}= & -A \cos \phi\left(\begin{array}{c}
v_{*}\left(2 r_{\epsilon \epsilon 0}+r_{\epsilon \epsilon 2}\right)-r_{*}\left(2 v_{\epsilon \epsilon 0}+v_{\epsilon \epsilon 2}\right) \\
\pi^{2} r_{*}\left(2 r_{\epsilon \epsilon 0}+r_{\epsilon \epsilon 2}\right)+v_{*}\left(2 v_{\epsilon \epsilon 0}+v_{\epsilon \epsilon 2}\right)
\end{array}\right) \\
& -A \cos (3 \phi)\left(\begin{array}{c}
v_{*} r_{\epsilon \epsilon 2}-r_{*} v_{\epsilon \epsilon 2} \\
\pi^{2} r_{*} r_{\epsilon \epsilon 2}+v_{*} v_{\epsilon \epsilon 2}
\end{array}\right) .
\end{aligned}
$$

Thus, the solvability condition gives

$$
\begin{gathered}
\mathbf{u}_{c}^{\dagger}\left(\begin{array}{c}
r_{*} \partial_{\tilde{T}} A \\
-v_{*} \partial_{\tilde{T}} A-\delta j_{1} r_{*} A
\end{array}\right) \\
=-A \mathbf{u}_{c}^{\dagger}\left(\begin{array}{c}
v_{*}\left(2 r_{\epsilon \epsilon 0}+r_{\epsilon \epsilon 2}\right)-r_{*}\left(2 v_{\epsilon \epsilon 0}+v_{\epsilon \epsilon 2}\right) \\
\pi^{2} r_{*}\left(2 r_{\epsilon \epsilon 0}+r_{\epsilon \epsilon 2}\right)+v_{*}\left(2 v_{\epsilon \epsilon 0}+v_{\epsilon \epsilon 2}\right)
\end{array}\right) .
\end{gathered}
$$

Substituting the coefficients (C11), (C12), (C13), and (C14) into Eq. (C16) gives the desired amplitude equation

$$
\partial_{\tilde{T}} A=\pi r_{*}^{2} \delta j_{1} A+\tilde{a} A^{3},
$$

where the parameter $\tilde{a}$ is

$$
\begin{aligned}
\tilde{a}= & \pi\left(5 v_{*}^{4}+\pi^{4} r_{*}^{4}-\frac{5}{2}\right)\left(\frac{1}{j_{1}^{T}-j_{0}}+\frac{1 / 2}{j_{1}^{T}-j_{2}}\right) \\
& -v_{*}\left(\frac{j_{0}}{j_{1}^{T}-j_{0}}+\frac{j_{2} / 2}{j_{1}^{T}-j_{2}}\right) .
\end{aligned}
$$

Equating Eq. (C18) to zero gives the critical boundary $j_{2}^{c}$ separating subcritical and supercritical Turing bifurcations:

$j_{2}^{c}=\frac{3 j_{1}^{T}-j_{0}}{2}+\frac{6\left(j_{1}^{T}-j_{0}\right)^{2} \pi^{2} r_{*}^{3}}{5+4 \pi^{2} r_{*}^{3}\left(3 j_{0}-j_{1}^{T}-10 \pi^{2} r_{*}+4 \pi^{6} r_{*}^{5}\right)}$.

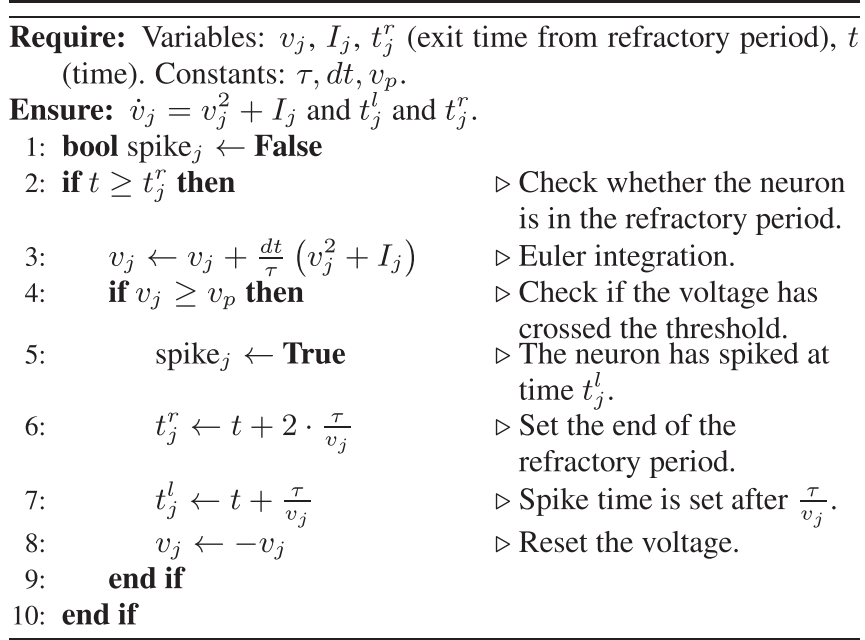

FIG. 6. Algorithm used for the Euler integration of the QIF neuron Eq. (2).

In dimensional form, Eqs. (C17), (C18), and (C19) are, respectively:

$$
\begin{aligned}
\tau \partial_{T} A=\pi \frac{\tau^{2} R_{*}^{2}}{\Delta} \delta J_{1} A+a A^{3}, & (\mathrm{C} 20) \\
a= & {\left[\pi\left(\frac{5 \Delta^{3}}{16 \pi^{4} \tau^{4} R_{*}^{4}}+\frac{\pi^{4} \tau^{4} R_{*}^{4}}{\Delta}-\frac{5 \Delta}{2}\right)\left(\frac{1}{J_{1}^{T}-J_{0}}+\frac{1 / 2}{J_{1}^{T}-J_{2}}\right)\right.} \\
+ & \left.\frac{\Delta}{2 \pi \tau R_{*}}\left(\frac{J_{0}}{J_{1}^{T}-J_{0}}+\frac{J_{2} / 2}{J_{1}^{T}-J_{2}}\right)\right],
\end{aligned}
$$

and

$$
\begin{aligned}
J_{2}^{c}= & \frac{3 J_{1}^{T}-J_{0}}{2} \\
& +\frac{6\left(J_{1}^{T}-J_{0}\right)^{2} \pi^{2} \tau^{3} R_{*}^{3}}{5 \Delta^{2}+4 \pi^{2} \tau^{3} R_{*}^{3}\left(3 J_{0}-J_{1}^{T}-10 \pi^{2} \tau R_{*}+\frac{4 \pi^{6} \tau^{5} R_{*}^{5}}{\Delta^{2}}\right.} .
\end{aligned}
$$

\section{APPENDIX D: NUMERICAL SIMULATIONS}

\section{Numerical simulation of the QIF model}

In numerical simulations, we used the Euler scheme with time step $d t=10^{-3}$. Additionally, we considered the peak and reset values $v_{p}=-v_{r}=100$. The algorithm used to simulate the QIF neuron (2) is shown in Fig. 6.

\section{Numerical simulation of the ring network}

To numerically implement the ring network of QIF neurons we divided the ring into $m=100$ intervals located at $\phi_{l}=2 \pi l / m-\pi$, where $l=1, \ldots, m$. At each location $\phi_{l}$, we considered $n=2.5 \times 10^{3}$ excitatory and $n=2.5 \times 10^{3}$ inhibitory neurons (i.e., the ring consisted of a total of $2 N=2 \mathrm{mn}=5 \times 10^{5}$ QIF neurons).

The neurons in each location $\phi_{l}$ receive Lorentziandistributed currents, which have been generated using the 
formula

$$
\eta_{i}=\bar{\eta}+\Delta \tan \left[\frac{\pi}{2} \frac{2 i-n-1}{n+1}\right], \quad i=1, \ldots, n .
$$

On the other hand, perturbations (applied at time $t_{0}$ ) are modeled using the function

$$
P^{e, i}(\phi, t)=A\left(e^{\left(t-t_{0}\right) / \tau_{r}}-1\right) \cdot \cos (K \cdot \phi),
$$

where $A$ is the amplitude, $K$ is the wave number, and $\tau_{r}$ is the rising time constant of the perturbation. In Figs. 2, 3, and 5 we used $t_{0}=0.05 \mathrm{~s}, A=0.3$, and $\tau_{r}=4 \times 10^{-3} \mathrm{~s}$. The perturbations had a duration of $0.01 \mathrm{~s}$.

Finally, the instantaneous firing rates in Fig. 2 are obtained binning time and counting the spikes of neurons in each interval $\phi_{l}$ within a sliding time window of size $\delta t=0.01 \mathrm{~s}$.
[1] H. R. Wilson and J. D. Cowan, A mathematical theory of the functional dynamics of cortical and thalamic nervous tissue, Kybernetik 13, 55 (1973).

[2] S.-I. Amari, A method of statistical neurodynamics, Kybernetik 14, 201 (1974).

[3] S.-I. Amari, Dynamics of pattern formation in lateral-inhibition type neural fields, Biol. Cybern. 27, 77 (1977).

[4] Paul L. Nunez, The brain wave equation: a model for the eeg, Math. Biosci. 21, 279 (1974).

[5] B. Ermentrout, Neural networks as spatio-temporal patternforming systems, Rep. Prog. Phys. 61, 353 (1998).

[6] S. Coombes, Waves, bumps, and patterns in neural field theories, Biol. Cybern. 93, 91 (2005).

[7] G. B. Ermentrout and D. H. Terman, Mathematical Foundations of Neuroscience, Vol. 64 (Springer, Berlin, 2010).

[8] Paul C. Bressloff, Spatiotemporal dynamics of continuum neural fields, J. Phys. A 45, 033001 (2012).

[9] S. Coombes, P. beim Graben, and R. Potthast, Tutorial on neural field theory, in Neural Fields (Springer, Berlin, 2014), pp. 1-43.

[10] G. Deco, V. K. Jirsa, P. A. Robinson, M. Breakspear, and K. Friston, The dynamic brain: From spiking neurons to neural masses and cortical fields, PLoS Comput. Biol. 4, e1000092 (2008).

[11] Hugh R. Wilson and Jack D. Cowan, Excitatory and inhibitory interactions in localized populations of model neurons, Biophys. J. 12, 1 (1972).

[12] B. Ermentrout, Reduction of conductance-based models with slow synapses to neural nets, Neur. Comput. 6, 679 (1994).

[13] N. Brunel and X.-J. Wang, What determines the frequency of fast network oscillations with irregular neural discharges? I. Synaptic dynamics and excitation-inhibition balance, J. Neurophysiol. 90, 415 (2003).

[14] David J. Pinto and G. Bard Ermentrout, Spatially structured activity in synaptically coupled neuronal networks: Ii. Lateral inhibition and standing pulses, SIAM J Appl. Math. 62, 226 (2001).

[15] V. K. Jirsa and H. Haken, A derivation of a macroscopic field theory of the brain from the quasi-microscopic neural dynamics, Physica D 99, 503 (1997).

[16] S. Coombes, G. J. Lord, and M. R. Owen, Waves and bumps in neuronal networks with axo-dendritic synaptic interactions, Physica D 178, 219 (2003).

[17] Fatihcan M. Atay and Axel Hutt, Stability and bifurcations in neural fields with finite propagation speed and general connectivity, SIAM J. Appl. Math. 65, 644 (2004).

[18] S. Coombes and C. Laing, Delays in activity-based neural networks, Philos. Trans. Roy. Soc. Lond. A 367, 1117 (2009).
[19] L. Zhang, How do synaptic coupling and spatial temporal delay influence traveling waves in nonlinear nonlocal neuronal networks? SIAM J. Appl. Dynam. Syst. 6, 597 (2007).

[20] A. Hutt, Local excitation-lateral inhibition interaction yields oscillatory instabilities in nonlocally interacting systems involving finite propagation delay, Phys. Lett. A 372, 541 (2008).

[21] J. Touboul, Mean-field equations for stochastic firing-rate neural fields with delays: Derivation and noise-induced transitions, Physica D 241, 1223 (2012).

[22] R. Veltz, Interplay between synaptic delays and propagation delays in neural field equations, SIAM J. Appl. Dynam. Syst. 12, 1566 (2013).

[23] K. Dijkstra, S. A. van Gils, S. G. Janssens, Yu. A. Kuznetsov, and S. Visser, Pitchforkhopf bifurcations in 1d neural field models with transmission delays, Physica D 297, 88 (2015).

[24] A. Roxin, N. Brunel, and D. Hansel, Role of Delays in Shaping Spatiotemporal Dynamics of Neuronal Activity in Large Networks, Phys. Rev. Lett. 94, 238103 (2005).

[25] D. Battaglia, N. Brunel, and D. Hansel, Temporal Decorrelation of Collective Oscillations in Neural Networks with Local Inhibition and Long-Range Excitation, Phys. Rev. Lett. 99, 238106 (2007).

[26] A. Roxin and E. Montbrió, How effective delays shape oscillatory dynamics in neuronal networks, Physica D 240, 323 (2011).

[27] E. S. Schaffer, S. Ostojic, and L. F. Abbott, A complex-valued firing-rate model that approximates the dynamics of spiking networks, PLoS Comput. Biol. 9, e1003301 (2013).

[28] E. Montbrió, D. Pazó, and A. Roxin, Macroscopic Description for Networks of Spiking Neurons, Phys. Rev. X 5, 021028 (2015).

[29] E. Ott and T. M. Antonsen, Low dimensional behavior of large systems of globally coupled oscillators, Chaos 18, 037113 (2008).

[30] E. Ott and T. M. Antonsen, Long time evolution of phase oscillator systems, Chaos 19, 023117 (2009).

[31] E. Ott, B. R. Hunt, and T. M. Antonsen, Comment on "long time evolution of phase oscillators systems,"Chaos 21, 025112 (2011).

[32] G. B. Ermentrout and N. Kopell, Parabolic bursting in an excitable system coupled with a slow oscillation, SIAM J. Appl. Math. 46, 233 (1986).

[33] E. M. Izhikevich, Dynamical Systems in Neuroscience (The MIT Press, Cambridge, MA, 2007).

[34] The stability analysis of the original Eqs. (6) gives two additional complex eigenvalues for each oscillatory mode $K$. These eigenvalues are degenerated and are associated to the oscillatory modes of the uncoupled neuronal system, that is they coincide with (10) with $J_{K}^{e, i}=0$. Additionally, due to the translational invariance of the SHS solutions, each of the 
eigenvalues Eq. (10) is two-times degenerated, corresponding to even and odd perturbations. See Appendix B for the detailed linear stability analysis of the QIF-NFM Eqs. (6).

[35] J. Rankin, D. Avitabile, J. Baladron, G. Faye, and David J B Lloyd, Continuation of localized coherent structures in nonlocal beural field equations, SIAM J. Sci. Comput. 36, B70 (2014).

[36] Daniele Avitabile, Numerical computation of coherent structures in spatially-extended systems, Second International Conference on Mathematical Neuroscience, Antibes Juan-les-Pins (2016), https://icmns2016.inria.fr/pre-conference-workshop/.

[37] T. B. Luke, E. Barreto, and P. So, Complete classification of the macroscopic behavior of a heterogeneous network of theta neurons, Neural Comput. 25, 3207 (2013).

[38] P. So, T. B. Luke, and E. Barreto, Networks of theta neurons with time-varying excitability: Macroscopic chaos, multistability, and final-state uncertainty, Physica D 267, 16 (2014).

[39] C. R. Laing, Derivation of a neural field model from a network of theta neurons, Phys. Rev. E 90, 010901 (2014).

[40] D. Pazó and E. Montbrió, Low-Dimensional Dynamics of Populations of Pulse-Coupled Oscillators, Phys. Rev. X 4, 011009 (2014).

[41] Kevin P. O'Keeffe and Steven H. Strogatz, Dynamics of a population of oscillatory and excitable elements, Phys. Rev. E 93, 062203 (2016).

[42] S. Chandra, D. Hathcock, K. Crain, T. M. Antonsen, M. Girvan, and E. Ott, Modeling the network dynamics of pulse-coupled neurons, Chaos 27, 033102 (2017).

[43] S. Coombes and Á. Byrne, Next generation neural mass models, in Lecture Notes in Nonlinear Dynamics in Computational Neuroscience: from Physics and Biology to ICT Springer (In Press).

[44] Á Byrne, M. J. Brookes, and S. Coombes, A mean field model for movement induced changes in the beta rhythm, J. Comput. Neurosci. 43, 143 (2017).

[45] R. Gallego, E. Montbrió, and D. Pazó, Synchronization scenarios in the Winfree model of coupled oscillators, Phys. Rev. E 96, 042208 (2017).
[46] D. Avitabile, M. Desroches, and E. Knobloch, Spatiotemporal canards in neural field equations, Phys. Rev. E 95, 042205 (2017).

[47] D. Pazó and E. Montbrió, From Quasiperiodic Partial Synchronization to Collective Chaos in Populations of Inhibitory Neurons with Delay, Phys. Rev. Lett. 116, 238101 (2016).

[48] I. Ratas and K. Pyragas, Macroscopic self-oscillations and aging transition in a network of synaptically coupled quadratic integrate-and-fire neurons, Phys. Rev. E 94, 032215 (2016).

[49] F. Devalle, A. Roxin, and E. Montbrió, Firing rate equations require a spike synchrony mechanism to correctly describe fast oscillations in inhibitory networks, arXiv:1705.09205 (2017).

[50] Carlo R Laing, Exact neural fields incorporating gap junctions, SIAM J. Appl. Dynam. Syst. 14, 1899 (2015).

[51] Carlo R. Laing, Travelling waves in arrays of delay-coupled phase oscillators, Chaos 26, 094802 (2016).

[52] Carlo R. Laing, Bumps in small-world networks, Front. Comput. Neurosci. 10, 53 (2016).

[53] Carlo R. Laing, The dynamics of chimera states in heterogeneous kuramoto networks, Physica D 238, 1569 (2009).

[54] Oleh E Omel'chenko, Coherence-incoherence patterns in a ring of non-locally coupled phase oscillators, Nonlinearity 26, 2469 (2013).

[55] E Omel'chenko, Matthias Wolfrum, and Carlo R. Laing, Partially coherent twisted states in arrays of coupled phase oscillators, Chaos 24, 023102 (2014).

[56] Yoji Kawamura, From the Kuramoto-Sakaguchi model to the Kuramoto-Sivashinsky equation, Phys. Rev. E 89, 010901 (2014).

[57] The derivation of the QIF-NFM Eq. (6) can be readily extended to account for populations of excitatory and inhibitory neurons of different sizes, i.e., $N^{e} \neq N^{i}$. This can be always achieved by rescaling the relative contributions of the excitatory and inhibitory mean fields as $S(\phi)=p^{e} S^{e}(\phi)+p^{i} S^{i}(\phi)$, where $p^{e}=2 N^{e} /\left(N^{e}+N^{i}\right)$ and $p^{i}=2 N^{i} /\left(N^{e}+N^{i}\right)$. In the case of the effective model Eq. (7), this implies that Fourier coefficients in Eq. (8) need to be changed as $J_{K}=p^{e} J_{K}^{e}-p^{i} J_{K}^{i}$.

[58] Y. Kuramoto, Chemical Oscillations, Waves, and Turbulence (Springer-Verlag, Berlin, 1984). 